\title{
Development of Monoclonal Antibody Based IgG and IgM ELISA for Diagnosis of Severe Fever with Thrombocytopenia Syndrome Virus Infection
}

\section{Mei Zhang}

Guizhou University https://orcid.org/0000-0001-9242-350X

\section{Yanhua Du}

Shanxi Province Disease Prevention and Control Center: Shanxi Center for Disease Control and

Prevention

Li Yang

Guizhou University

\section{Lin Zhan}

Guizhou Provincial People's Hospital

\section{Bin Yang}

Guizhou Provincial People's Hospital

\section{Xueyong Huang}

Henan Province Center for Disease Control and Prevention

\section{Bianli Xu}

Henan Province Center for Disease Control and Prevention

\section{Koichi Morita}

Nagasaki University: Nagasaki Daigaku

Fuxun Yu ( $\square$ yufuxun@126.com )

https://orcid.org/0000-0001-8804-2755

\section{Research}

Keywords: Severe fever with thrombocytopenia syndrome virus, Recombinant nucleocapsid protein, Monoclonal antibody

Posted Date: December 30th, 2021

DOI: https://doi.org/10.21203/rs.3.rs-1199065/v1

License: (c) (1) This work is licensed under a Creative Commons Attribution 4.0 International License. Read Full License 


\section{Abstract}

Background: Severe fever with thrombocytopenia syndrome virus (SFTSV) is a newly emerged virus that possesses a great threat to human health because of the high fatality rate.

Method: To develop sensitive and specific sero-diagnosis systems for SFTSV infections, monoclonal antibodies (MAbs) against recombinant SFTSV nucleocapsid (rSFTSV-N) protein were developed by immunizing BALB/C mice with rSFTSV-N protein and fusing the spleen cells with SP2/0 myeloma cells. Three hybridoma cell lines secreting MAbs against rSFTSV-N were obtained. MAb based IgG sandwich enzyme linked immunosorbent assay (ELISA) and IgM capture ELISA systems were established by using the newly developed MAbs. One hundred fifteen clinical suspected SFTS patient serum samples were used to evaluate the newly established systems by comparing with the total antibody detecting sandwich ELISA system and indirect ELISA systems.

Results: The MAb based sandwich IgG ELISA was perfectly matched with that of the total antibody sandwich ELISA and the indirect IgG ELISA with a sensitivity and specificity of $100 \%$. IgM capture ELISA results perfectly matched with that of the total antibody sandwich ELISA while was more sensitive comparing with the indirect IgM ELISA.

Conclusions: The MAbs against rSFTSV-N protein offer new tools for SFTSV studies and our newly developed MAb-based IgG and IgM capture ELISA systems would offer safe and useful tools for diagnosis of SFTS virus infections and epidemiological investigations.

\section{Background}

Severe fever thrombocytopenia syndrome (SFTS) is an emerging, tick-borne infectious disease caused by SFTS virus, characterized by high fever, leukopenia, thrombocytopenia, and multiple organ dysfunctions, including the lung, liver, kidney and so forth [1-3]. SFTSV is recognized as Phlebovirus genus in the Bunyaviridae family, with a high mortality up to $30 \%$ at early epidemic period [3, 4]. Since the first report of SFTS in China, the incidence of SFTS has expanded to at least 15 provinces in China; and other Asian countries such as South Korea, Japan and Vietnam have also discovered this disease one after another, indicating that the epidemic area is extending [5-7]. Therefore, due to the worldwide spread of SFTSV, high mortality rate and human communicable nature of the virus, the World Health Organization (WHO) has listed the SFTS virus as one of the most in need of attention pathogens [8]. Nonetheless, to date, there are no available effective antiviral drugs or vaccines against SFTSV, and more diagnostic tools for detecting SFTSV are needed [9].

Using clinical manifestation to diagnose SFTSV is non-specific, since SFTS is difficult to distinguish from many other diseases with similar clinical features [6]. Although virus isolation from blood of viremic patients is a reliable method to diagnose SFTSV infection, this way is time-consuming and requires high security biocontainment facility $[10,11]$. There are several methods to detect SFTSV genome. The reverse transcription polymerase chain reaction (RT-PCR) [11-13], quantitative real-time RT-PCR [11, 14, 15], and 
reverse transcription loop-mediated isothermal amplification assay (RT-LAMP) [16-18] have been used for detecting SFTSV genome. Studies have shown that these methods are only suitable for acute onset period of 1-6 days $[1,10,19]$. Hence, complement methods for the diagnosis of SFTSV are essential.

SFTSV is a single-stranded, negative RNA virus with three segments, designated $L, M$, and $S$ [20]. The $L$ segment encodes the RNA-dependent RNA polymerase (RaRp); the M segment encodes the glycoprotein precursor (GCP), including $\mathrm{Gc}$ and Gn glycoprotein; and the S segment encodes nucleocapsid protein (NP) and nonstructural protein (NSs) via using ambisense coding [21, 22]. NP is highly conserved and immunogenic in Bunyaviridae family. It has been shown that NP plays an important role in the virus replication, transcription, packaging of genomic RNA into ribonucleoprotein [23,24]. Our previous study demonstrated that recombinant SFTSV nucleocapsid (rSFTSV-N) protein based indirect ELISA assay systems has been established to detect specific human IgG and IgM antibodies, respectively. However, rSFTSV-N protein based indirect IgM ELISA missed to detect several patients [25].

In the present study, three monoclonal antibodies (MAbs: 5G12, 4A10, 1C3) against rSFTSV-N protein were successfully developed, and the MAb based IgG sandwich ELISA and IgM capture ELISA system were established. Serum samples, collected from clinically-suspected SFTS patients, were used to evaluate the newly established systems and results were compared with the total antibody detecting sandwich ELISA system and the indirect ELISA systems.

\section{Materials And Methods Serum samples}

To evaluate the MAb based IgG sandwich ELISA and IgM capture ELISA system, 115 serum samples collected from Henan Province, China, which were collected from patients who recovered from suspected SFTS disease were used.

\section{BALB/C mice immunization}

The rSFTSV-N protein expressed and purified as previously reported [25] was emulsified with equal volumes of Freund's complete adjuvant (MP Biochemicals, CA, USA) and injected intraperitoneally into three 6 week old female BALB/c mice (Chongqing Tengxin Bill Experimental Animal Sales Co., LTDChongqing, China), at a dose of $100 \mu \mathrm{g}$ per injection. On day 14 , all mice were boosted with rSFTSV-N protein in equal volumes Freund's incomplete adjuvant (MP Biochemicals, CA, USA). Ten days after the second immunizations, blood samples were collected for antibody titer measurement via indirect ELISA. On day $28,29,30$, mice were injected intraperitoneally with $100 \mu \mathrm{g}$ /day rSFTSV-N protein for 3 times. On day 31 , blood samples were collected and the spleen was taken out for cell fusion.

\section{Preparation of monoclonal antibodies against rSFTSV-N protein}


After immunization, the spleen cells were collected for cell fusion with SP2/0 myeloma cells (from the previous experiment) [26] using polyethylene glycol (PEG 1500, Roche, Indiana, USA). The hybridoma cells were grown in a selective medium of hypoxanthine aminopterin - thymidine (HAT) (Gibco, NY, USA) for 10 days and then screened by indirect ELISA to select cells producing antibody against rSFTSV-N protein. The positive clones were diluted to establish single stable clones by limited dilution. In short, hybridoma cell suspension was diluted in growth medium (RPMI 1640, Gibco, NY, USA) supplemented with $10 \%$ fetal bovine serum (FBS) and inoculated to 96-well microplates with approximately 1 cell/well for 10 days before performing indirect ELISA to select clones secreting the desired antibody.

Subsequently, the positive clones were transferred to culture flasks and propagated in growth media. At last, large-scale production of MAbs were done by propagate positive clones in Hybridoma-SFM medium (Gibco, NY, USA) and injected hybridoma cells intraperitoneally into mouse to produce ascetic fluid. MAb HiTrap protein G chromatograph kit (GE Healthcare, Uppsala, Sweden) was applied to purify MAbs.

\section{Screening of MAbs by ELISA}

The presence of antibodies in the supernatant of hybridoma cells culture media were assessed by ELISA according our previously published protocols [26] with the following modification. The 96-well plates were processed as follows: coating of $50 \mathrm{ng} /$ well rSFTSV-N protein at $4^{\circ} \mathrm{C}$ overnight, then blocking using PBST with $5 \%$ nonfat milk, at room temperature for $1 \mathrm{~h}$ followed by adding hybridoma culture supernatant or adding pre-immunization and post immunization mice serum samples for negative and positive controls, respectively, at $37^{\circ} \mathrm{C}$ for $1 \mathrm{~h}$; followed by the detection of bound IgG with horseradish-peroxidaseconjugated (HRP) goat anti-mouse IgG (American Qualex, San Clemente, CA) diluted $1: 10,000$ at $37^{\circ} \mathrm{C}$ for 1h. Then $\mathrm{H}_{2} \mathrm{O}_{2}$-ABTS [2,2' azinobis (3-ethylbenzthiazolinesulfonic acid)] substrate was added and optical density (OD) value was recorded. All clones with $O D$ value twice or higher than that of the negative control were considered positive.

\section{Immunofluorescence analysis}

Indirect immunofluorescence test was applied to determine the reactivity of MAbs with SFTSV, SFTSVinfected Vero-E6 (from the previous experiment) [26] were collected and centrifuged at $600 \mathrm{xg}$ for 5 minutes, and then, the cell pellet was washed 3 times with PBS and spotted onto the Teflon-coated 8multi-well glass slide (MP Biochemicals, CA, USA). After the slide was air dried, cells were fixed in cold acetone at $4^{\circ} \mathrm{C}$ for $10 \mathrm{~min}$. After blocking with BlockAce for $30 \mathrm{~min}$ at room temperature, the cells were incubated with $15 \mu \mathrm{l}$ culture media of the hybridoma cells in a wet chamber at $37^{\circ} \mathrm{C}$ for $1 \mathrm{~h}$. Then, the slides were washed and air-dried once more, followed by detection using $15 \mu \mathrm{l}$ of fluorescein isothiocyanate (FITC) conjugated goat anti-mouse IgG (Bethyl Laboratories Inc. Montgomery, USA) at a dilution of $1: 50$ to every test well and reacting in dark at $37^{\circ} \mathrm{C}$ for $1 \mathrm{~h}$. Finally, the slides were washed and sealed with mounting medium for fluorescence (VectorLaboratories, Inc.). The images were acquired using an OLYMPUS IX73 immunofluorescence microscope.

\section{Western blotting}


Western blot analysis was performed as described before [25]. Briefly, protein molecular weight marker (PageRuler ${ }^{\mathrm{TM}}$ Prestained Protein Ladder, Thermo Scientific, Denmark) and the purified rSFTSV-N protein were separated in a $15 \%$ polyacrylamide gel. The separated proteins were transferred onto a polyvinylidene fluoride (PVDF) microporous membrane (Immobilon, Millipore, USA). The membrane was first blocked and then incubated at $4^{\circ} \mathrm{C}$ overnight or $37^{\circ} \mathrm{C}$ for 1 hour with the supernatant of hybridoma cells, followed by a secondary antibody of goat anti-mouse lgG (American Qualex, Califonia, USA, 1:1000 dilution). Protein bands were visualized using Metal Enhanced dimethyl aminobenzidine (DAB) Substrate Kits (Solarbio, China).

\section{Detection of MAbs isotypes}

The MAb isotype was determined by the SBA Clonotyping System-HRP kit according to the manufacturer's instructions (Southern Biotech, USA).

\section{ELISA procedures for human serum}

To evaluate MAbs for diagnosis of SFTSV infection, we established MAb-based IgG sandwich ELISA and IgM capture ELISA system for human sera. These ELISA systems that possess common procedures was described here and the procedures specific for each assay will described under each assay.

Ninety-six well Nunc immunoplates (Thermo Scientific, Denmark) were used with a sample volume of 100 $\mu \mathrm{L} /$ well. The coating buffer was $0.01 \mathrm{M}$ PBS, $\mathrm{pH} 7.4$, and the plate coating was done at $4^{\circ} \mathrm{C}$ overnight. After exposure to a specific reagent at each step of the system except at the last step as described below, plates were washed three times with wash buffer (0.01 M PBS with $0.1 \%$ (vol/vol) Tween 20 (PBS-T)). PBS-T with $5 \%$ nonfat milk (Difco, Detroit, USA) was used to dilute all serum samples and reagents. In addition to substrate, all incubations were done at $37^{\circ} \mathrm{C}$ for $1 \mathrm{~h}$. Besides, plates with $100 \mu \mathrm{L} / \mathrm{well}^{\mathrm{H}} \mathrm{H}_{2} \mathrm{O}_{2}{ }^{-}$ ABTS substrate (Kirkegaatrd \& Perry, Gaithersburg, MD) were incubated at $37^{\circ} \mathrm{C}$ for $30 \mathrm{~min}$. A spectrophotometer was used to read the plates and record the OD values at $410 \mathrm{~nm}$.

\section{MAb-based IgG sandwich ELISA}

In the MAb-based IgG sandwich ELISA, 96-well plates were processed as follows: coating of $50 \mathrm{ng} /$ well of MAb overnight before blocking using PBS-T with $5 \%$ nonfat milk for $1 \mathrm{~h}$, followed by $50 \mathrm{ng} /$ well rSFTSV-N protein, then human serum samples diluted at 1:1,000 in 5\% nonfat milk in PBS-T were added, afterwards detection of bound IgG with HRP-goat anti-human IgG (American Qualex, Califonia, USA) diluted at 1:30,000, which was made visible after adding $\mathrm{H}_{2} \mathrm{O}_{2}$-ABTS substrate. OD values measured at $410 \mathrm{~nm}$ using a microplate spectrophotometer. Each serum specimen was tested twice and the average OD value was calculated. Each test used positive and negative control serum sample. The mean OD value of a sample more than twice the mean OD of the negative control serum was considered positive.

\section{MAb-based IgM capture ELISA}

The MAb-based IgM capture ELISA followed the steps below. Firstly, for each serum sample to be tested, 4 wells were coated with 1:500 dilution of anti-human IgM (Cappel, MP Biochemicals) before blocking 
using PBS-T with $5 \%$ nonfat milk for $1 \mathrm{~h}$. Secondly, 1:400 diluted human serum was added to the 4 wells. Afterwards two wells were added with $50 \mathrm{ng}$ of rSFTSV-N protein (positive antigen) and other two wells were added with $50 \mathrm{ng}$ of nonreactive protein (negative antigen). And then, $100 \mu \mathrm{L} /$ well of purified MAb diluted at 1:10,000 was added to all four wells, after which a 1:10,000 diluted peroxidase conjugated antimouse IgG (American Qualex, Califonia, USA) was then added. Finally, the remaining steps for colour development were the same as above.

\section{Total antibody ELISA and indirect IgG, IgM ELISA}

Detection of total antibody against SFTSV was done using a commercial ELISA kit (Xinlianxin Biomedical Technology CO., LTD, Wuxi, Jiangsu, China) following the manufacturer's instructions. Indirect IgG and IgM ELISA were done as previously described [25].

\section{Result}

\section{Preparation of monoclonal antibodies against rSFTSV-N protein}

BALB/c mice were immunized with rSFTSV-N protein 5 times with an immunization regimen as shown in Figure 1A. After subcloning and multiple rounds of indirect ELISA screening, 3 positive hybridoma clones producing MAbs against rSFTSV-N protein (designated as 5G12, 4A10, 1C3) were obtained.

Indirect immunofluorescence test showed strong fluorescence in Vero-E6 cells infected with SFTSV, no fluorescence was observed in mock Vero-E6 cells (Figure 1B). Western blot assay showed that these three MAbs all reacted with rSFTSV-N protein (Figure 2A). The classes of three MAbs were determined to be IgG2 $b$ and $\mathrm{k}$ chain (Figure 2B). These results confirmed that the 3 hybridoma cells secreted MAbs specific for SFTSTV.

\section{Evaluation of MAb-based IgG sandwich ELISA}

To evaluate the possibility of using the newly developed MAbs for diagnosis, MAb-based IgG sandwich ELISA for human serum was established. Among the 115 human serum samples from SFTS suspected patients, 85 samples were IgG positive and 30 were IgG negative by the IgG sandwich ELISA system. These results perfectly matched the total antibody sandwich ELISA and the rSFTSV-N protein based indirect IgG ELISA results. The sensitivity and specificity of MAb-based IgG sandwich ELISA were $100 \%$ and with a $100 \%$ concordance to these 2 ELISA systems (Table 1 and Table 2). 
Table 1

Comparation of MAb-based IgG sandwich ELISA with total antibody sandwich ELISA

\section{Total antibody \\ sandwich ELISA}

Positive

Negative

Total
MAb-based IgG sandwich ELISA

Negative

Positive

85

0

85
0

30

30
Total

85

30

115

Concordance $^{\mathrm{a}}$ : 100\% Sensitivity ${ }^{\mathrm{b}}$ : 100\% Specificityc: $100 \%$

a (No. of samples positive by both methods + No. of samples negative by both methods)/total number of samples $\times 100$

b True positive/(true positive + false negative) $\times 100$

${ }^{\mathrm{c}}$ True negative/(true negative + false positive) $\times 100$

\section{Table 2}

Comparation of MAb-based IgG sandwich ELISA with rSFTSV-N protein based indirect IgG ELISA

\section{MAb-based IgG sandwich ELISA}

rSFTSV-N-IgG indirect ELISA

Total

\section{Positive}

85

0

Negative

Total
85

\section{Negative}

0

30

30

115

Concordance $^{\text {a. }}$ : 100\% Sensitivity ${ }^{\text {b: }}$ 100\% Specificityc: $100 \%$

a (No. of samples positive by both methods + No. of samples negative by both methods)/total number of samples $\times 100$

${ }^{b}$ True positive/(true positive + false negative) $\times 100$

${ }^{c}$ True negative/(true negative + false positive $) \times 100$

\section{Evaluation of MAb based IgM capture ELISA}

IgM capture ELISA for human serum was established using the MAb as detecting antibody. Among the 115 human serum samples from SFTS-suspected patients, 85 samples were lgM positive and 30 were IgM negative by the IgM capture ELISA system which perfectly matched the total antibody sandwich ELISA results. The sensitivity and specificity of the IgM capture ELISA system were $100 \%$ with a $100 \%$ concordance to the total antibody sandwich ELISA system (Table 3). 
Table 3

Comparation of MAb-based IgM capture ELISA with total antibody sandwich ELISA

\section{Total antibody}

sandwich ELISA

Positive

Negative

Total
MAb-based IgM capture ELISA

Positive

85

0

85
Negative

0

85

30

30

30

Total

Concordance $^{\mathrm{a}}$ : $100 \%$ Sensitivity ${ }^{\mathrm{b}}: 100 \%$ Specificityc: $100 \%$

a (No. of samples positive by both methods + No. of samples negative by both methods)/total number of samples $\times 100$

${ }^{b}$ True positive/(true positive + false negative) $\times 100$

${ }^{c}$ True negative/(true negative + false positive) $\times 100$

Compared with the rSFTSV-N protein based indirect IgM ELISA, among the 85 positive samples detected by IgM capture ELISA, indirect IgM ELISA detected 77 positive, missing 8 positive samples. The concordance of the 2 methods was $93.04 \%$ with a sensitivity of $90.59 \%$ and specificity of $100 \%$ (Table 4).

Table 4

Comparation of MAb-based IgG sandwich ELISA with rSFTSV-N protein based indirect IgM ELISA

MAb-based IgM capture ELISA

rSFTSV-N-IgM indirect ELISA

Total

Positive

77

0

77

0

\section{Negative}

8

85

30

Total
30

38

Concordance $^{\text {a. }}$ 93.04\% Sensitivity ${ }^{\text {b: }}$ 90.59\% Specificity ${ }^{\text {c. }}$ 100\%

a (No. of samples positive by both methods + No. of samples negative by both methods)/total number of samples $\times 100$

${ }^{b}$ True positive/(true positive + false negative) $\times 100$

${ }^{c}$ True negative/(true negative + false positive) $\times 100$

\section{Disscussion}

Severe fever with thrombocytopenia syndrome is an acute viral infection mainly transmitted through tick bites, and person-to-person transmission is reported [27]. SFTSV has generated great concern as SFTS 
cases and related deaths has been on the rise with the high fatality rates of $16.4-30 \%$ [28]. For the diagnosis of SFTS, clinical manifestations of SFTS are non-specific, and as the direct evidence of SFTS infection, virus isolation from SFTSV-infected patients is time-consuming and needs highly safe biological protection facilities $[11,19]$. Therefore, safe methods with high sensitivity and specificity for the diagnosis of SFTSV are indispensable.

Due to the highly immunogenicity and abundance of NP in viral particles and infected cells, the recombinant NP is appropriate for using as a diagnostic antigen $[10,23,25]$. Because of the high purity and strong specificity, monoclonal antibodies can improve the sensitivity and specificity of various serological methods for detecting antigens $[26,29,30]$. MAbs against rSFTSV-NP for the diagnosis of SFTS has been documented for antigen detection and bovine serum antibody detection [31-33], but there is no report for application in detecting human antibodies. Detecting IgG or IgM antibodies in sera is a noted method for confirming infections, and studies have shown that IgM antibody detection is suitable for the early diagnosis of infection $[12,34,35]$.

Using the immunization schedule shown in Figure 1A, we used purified rSFTSV-N protein to immunize mice, and extracted the spleen cells for cell fusion with SP2/0 to prepare hybridoma cell lines secreting anti-rSFTSV-N protein MAbs. Three hybridoma cell lines secreting MAbs, named 5G12, 4A10, 1C3, with strong and specific reactivity to SFTSV were obtained. The MAbs reacted with the rSFTSV-N protein in indirect ELISA, immunofluorescence assay (Figure 1B) and Western blot (Figure 2A). These MAbs against SFTSV offer useful tool for the study of SFTSV.

MAb-based IgG sandwich ELISA and IgM capture ELISA to detect human serum antibodies against SFTSV were established and compared with the total antibody detecting sandwich ELISA system, indirect IgG and indirect IgM systems, respectively. Serum samples of 115 suspected SFTSV patients were evaluated, and the coincidence rates of MAb-based IgG sandwich ELISA or IgM capture ELISA with a total antibody detection sandwich ELISA and the indirect IgG ELISA were all 100\%, indicating that the newly developed MAb based ELISA systems are sensitive and specific methods for detecting SFTSV antibodies (Table 1, 2 and 3).

The IgM capture ELISA detected 8 more positive samples missed by the indirect IgM ELISA, confirmed that IgM capture ELISA is a more sensitive method for detecting $\lg \mathrm{M}$, probably because the $\lg \mathrm{M}$ capture ELISA eliminated the competed binding of IgG to antigen with the IgM [25, 36-38].

We have successfully demonstrated the sensitivity and specificity of the two MAbs-based ELISA systems, which do not require high-level microbiological safety facilities to prepare and use virus hence safe methods. The new systems detect IgG or IgM antibody separately, thereby they can distinguish previous or recent infection, thus provide more useful tools for SFTSV-infection diagnosis and epidemiological investigations.

\section{Conclusion}


Three MAbs against rSFTSV-N protein were successfully developed and used to develop MAb-based IgG sandwich ELISA and IgM capture ELISA systems for human serum. The two systems are safe, and have high sensitivity, specificity for the diagnosis of SFTS.

\section{Abbreviations}

\section{SFTSV}

Severe fever with thrombocytopenia syndrome virus

WHO

World Health Organization

RT-PCR

Reverse transcription polymerase chain reaction

RT-LAMP

Reverse transcription loop-mediated isothermal amplification assay

RaRp

RNA-dependent RNA polymerase

GCP

Glycoprotein precursor

NP

Nucleocapsid protein

NSs

Nonstructural protein

rSFTSV-N

Recombinant SFTSV nucleocapsid

ELISA

Enzyme linked immunosorbent assay

MAbs

Monoclonal antibodies

HAT

Hypoxanthine aminopterin-thymidine

FBS

Fetal bovine serum

HRP

Horseradish-peroxidase-conjugated

OD

Optical density

PBS

Phosphate buffered saline solution

PVDF

Polyvinylidene fluoride 
$\mathrm{DAB}$

Dimethyl aminobenzidine.

\section{Declarations}

\section{Acknowledgments}

We would like to thank the members of the Central Laboratory of Pulmonary Immunological Disease Research Centre, Chinese Health Ministry for their technical assistance and helpful discussions. We also acknowledged the School of Medicine, Guizhou University, for the training and support.

\section{Author Contributions}

M.Z., F.Y., B.Y., K.M.: Study design; M.Z., L.Y., X.H.: Laboratory experiments; M.Z., Y.D., L.Z., B.X.: Data analysis; M.Z., F.Y.: Writing of the manuscript. All authors have read and agreed to the published the final manuscript.

\section{Funding}

This work was supported by the National Natural Science Foundation of China ((2017) 81760605); the doctor's funding of Guizhou Provincial People's Hospital GSYSBS [2016]01.

\section{Availability of data and materials}

All relevant information is reflected in this current manuscript.

\section{Ethics approval and consent to participate}

This study was approved by the Ethical Committee of the Henan Provincial Center for Disease Control and Prevention (2016-KY-002-02). All experiments are carried out in compliance with approved guidelines and regulations. Part of this experiment used the remaining samples from the previous experiment. All serum sample providers have written informed consent to use their serum samples for research purposes.

\section{Consent for publication}

Not applicable.

\section{Competing interests}

The authors declare no conflict of interest.

\section{References}

1. Liu Q, He B, Huang SY, Wei F, Zhu XQ. Severe fever with thrombocytopenia syndrome, an emerging tick-borne zoonosis. Lancet Infect Dis. 2014;14:763-72. 
2. Zhang Y, Zhou D, Qin X, Tian J, Xiong Y, Wang J, et al. The ecology, genetic diversity, and phylogeny of Huaiyangshan virus in China. J Virol. 2012;86:2864-8.

3. Jin K, Koh Y, Ahn S, Cho J, Lim J, Song J, et al. Hard Ticks as Vectors Tested Negative for Severe Fever with Thrombocytopenia Syndrome in Ganghwa-do, Korea during 2019-2020. Korean J Parasitol. 2021;59:281-9.

4. Yu X, Liang M, Zhang S, Liu Y, Li J, Sun Y, et al. Fever with thrombocytopenia associated with a novel bunyavirus in China. N Engl J Med. 2011;364:1523-32.

5. Robles N, Han H, Park S, Choi Y. Epidemiology of severe fever and thrombocytopenia syndrome virus infection and the need for therapeutics for the prevention. Clin Exp Vaccine Res. 2018;7:43-50.

6. Seo J, Kim D, Yun N, Kim D. Clinical Update of Severe Fever with Thrombocytopenia Syndrome. Viruses. 2021;13:1213.

7. Sharma D, Kamthania M. A new emerging pandemic of severe fever with thrombocytopenia syndrome (SFTS). VirusDis. 2021;32:1-8.

8. Mehand M, Al-Shorbaji F, Millett P, Murgue B. The WHO R\&D Blueprint: 2018 review of emerging infectious diseases requiring urgent research and development efforts. Antiviral Res. 2018;159:63-7.

9. Li H, Zhang L, Li S, Zhang S, Wan W, Zhang Y, et al. Calcium channel blockers reduce severe fever with thrombocytopenia syndrome virus (SFTSV) related fatality. Cell Res. 2019;29:739-53.

10. Umeki K, Yasuda A, Umekita K, Megumi R, Nomura H, Kawaguchi T, et al. Detection of anti-SFTSV nuclear protein antibody in the acute phase sera of patients using double-antigen ELISA and immunochromatography. J Virol Methods. 2020;285:113942.

11. Matsuu A, Hamakubo E, Yabuki M. Seroprevalence of severe fever with thrombocytopenia syndrome virus in animals in Kagoshima Prefecture, Japan, and development of Gaussia luciferase immunoprecipitation system to detect specific IgG antibodies. Ticks Tick Borne Dis. 2021;12:101771.

12. Park E, Fujita O, Kimura M, Hotta A, Imaoka K, Shimojima M, et al. Diagnostic system for the detection of severe fever with thrombocytopenia syndrome virus RNA from suspected infected animals. PLoS One. 2021;16:e0238671.

13. Yoshikawa T, Fukushi S, Tani H, Fukuma A, Taniguchi S, Toda S, et al. Sensitive and specific PCR systems for detection of both Chinese and Japanese severe fever with thrombocytopenia syndrome virus strains and prediction of patient survival based on viral load. J Clin Microbiol. 2014;52:332533.

14. Lv SM, Yuan C, Zhang L, Wang YN, Dai ZN, Yang T, et al. Severe fever with thrombocytopenia syndrome with re-infection in China: a case report. Infect Dis Poverty. 2021;10:90.

15. Li Z, Qi X, Zhou M, Bao C, Hu J, Wu B, et al. A two-tube multiplex real-time RT-PCR assay for the detection of four hemorrhagic fever viruses: severe fever with thrombocytopenia syndrome virus, Hantaan virus, Seoul virus, and dengue virus. Arch Virol. 2013;158:1857-63.

16. Lee J, Won Y, Kang L, Lee S, Park S, Paik S. Development of a real-time loop-mediated isothermal amplification method for the detection of severe fever with thrombocytopenia syndrome virus. $J$ Microbiol. 2020;58:711-5.

Page $12 / 16$ 
17. Sano S, Fukushi S, Yamada S, Harada S, Kinoshita H, Sugimoto S, et al. Development of an RT-LAMP Assay for the Rapid Detection of SFTS Virus. Viruses. 2021;13:693.

18. Baek Y, Cheon H, Park S, Lloren K, Ahn S, Jeong J, et al. Simple, Rapid and Sensitive Portable Molecular Diagnosis of SFTS Virus Using Reverse Transcriptional Loop-Mediated Isothermal Amplification (RT-LAMP). J Microbiol Biotechnol. 2018;28:1928-36.

19. Ding Y, Liang M, Ye J, Liu Q, Xiong C, Long B, et al. Prognostic value of clinical and immunological markers in acute phase of SFTS virus infection. Clin Microbiol Infect. 2014;20:0870-8.

20. Brennan B, Li P, Zhang S, Li A, Liang M, Li D, et al. Reverse genetics system for severe fever with thrombocytopenia syndrome virus. J Virol. 2015;89:3026-37.

21. Yamada H, Taniguchi S, Shimojima M, Tan L, Kimura M, Morinaga $Y$, et al. M Segment-Based Minigenome System of Severe Fever with Thrombocytopenia Syndrome Virus as a Tool for Antiviral Drug Screening. Viruses. 2021;13:1061.

22. Zhang L, Fu Y, Zhang R, Guan Y, Jiang N, Zheng N, et al. Nonstructural Protein NSs Hampers Cellular Antiviral Response through LSm14A during Severe Fever with Thrombocytopenia Syndrome Virus Infection. J Immunol. 2021;207:590-601.

23. Wang G, Chang H, Jia B, Liu Y, Huang R, Wu W, et al. Nucleocapsid protein-specific IgM antibody responses in the disease progression of severe fever with thrombocytopenia syndrome. Ticks Tick Borne Dis. 2019;10:639-46.

24. Duan Y, Wu W, Zhao Q, Liu S, Liu H, Huang M, et al. Enzyme-Antibody-Modified Gold Nanoparticle Probes for the Ultrasensitive Detection of Nucleocapsid Protein in SFTSV. Int J Environ Res Public Health. 2020;17:4427.

25. Yu F, Du Y, Huang X, Ma H, Xu B, Adungo F, et al. Application of recombinant severe fever with thrombocytopenia syndrome virus nucleocapsid protein for the detection of SFTSV-specific human IgG and IgM antibodies by indirect ELISA. Virol J. 2015;12:117.

26. Adungo F, Yu F, Kamau D, Inoue S, Hayasaka D, Posadas-Herrera G, et al. Development and Characterization of Monoclonal Antibodies to Yellow Fever Virus and Application in Antigen Detection and IgM Capture Enzyme-Linked Immunosorbent Assay. Clin Vaccine Immunol. 2016;23:689-97.

27. Yoo J, Heo S, Park D, Kim H, Fukuma A, Fukushi S, et al. Family Cluster Analysis of Severe Fever with Thrombocytopenia Syndrome Virus Infection in Korea. Am J Trop Med Hyg. 2016;95:1351-7.

28. Yoo J, Kim J, Heo S, Kim J, Park H, Lee J, et al. Neutralizing Antibodies to Severe Fever With Thrombocytopenia Syndrome Virus Among Survivors, Non-Survivors and Healthy Residents in South Korea. Front Cell Infect Microbiol. 2021;11:649570.

29. Han D, Xu Y, Zhao X, Mao Y, Kang Q, Wen W, et al. A novel human anti-TIGIT monoclonal antibody with excellent function in eliciting NK cell-mediated antitumor immunity. Biochem Biophys Res Commun. 2021;534:134-40.

30. Sivaccumar J, Leonardi A, laccarino E, Corvino G, Sanguigno L, Chambery A, et al. Development of a New Highly Selective Monoclonal Antibody against Preferentially Expressed Antigen in Melanoma 
(PRAME) and Identification of the Target Epitope by Bio-Layer Interferometry. Int J Mol Sci. 2021;22:3166.

31. Zhuge Y, Ding C, Gong X, Hu D, Zhu J, Wang CJJjoid. Development and evaluation of two different double-antibody sandwich ELISAs for detecting severe fever with thrombocytopenia syndrome virus infection. Jpn J Infect Dis. 2021:JJID-2020.

32. Lee H, Kim E, Song J, Choi J, Lee J, Cho I, et al. Development and evaluation of a competitive enzyme-linked immunosorbent assay using a monoclonal antibody for diagnosis of severe fever with thrombocytopenia syndrome virus in bovine sera. J Vet Sci. 2016;17:307-14.

33. Fukuma A, Fukushi S, Yoshikawa T, Tani H, Taniguchi S, Kurosu T, et al. Severe Fever with Thrombocytopenia Syndrome Virus Antigen Detection Using Monoclonal Antibodies to the Nucleocapsid Protein. PLoS Negl Trop Dis. 2016;10:e0004595.

34. Du Y, Cheng N, Li Y, Wang H, You A, Su J, et al. Seroprevalance of antibodies specific for severe fever with thrombocytopenia syndrome virus and the discovery of asymptomatic infections in Henan Province, China. PLoS Negl. Trop Dis. 2019;13:e0007242.

35. Hu J, Shi C, Li Z, Guo X, Qian Y, Tan W, et al. A cluster of cases of severe fever with thrombocytopenia syndrome bunyavirus infection in China, 1996: A retrospective serological study. PLoS Negl Trop Dis. 2018;12:e0006603.

36. Otsuka N, Gotoh K, Nishimura N, Ozaki T, Nakamura Y, Haga K, et al. A Novel IgM-capture enzymelinked immunosorbent assay using recombinant Vag8 fusion protein for the accurate and early diagnosis of Bordetella pertussis infection. Microbiol Immunol. 2016;60:326-33.

37. L'Huillier A, Hamid-Allie A, Kristjanson E, Papageorgiou L, Hung S, Wong C, et al. Evaluation of Euroimmun Anti-Zika Virus IgM and IgG Enzyme-Linked Immunosorbent Assays for Zika Virus Serologic Testing. J Clin Microbiol. 2017;55:2462-71.

38. Emmerich P, Mika A, von Possel R, Rackow A, Liu Y, Schmitz H, et al. Sensitive and specific detection of Crimean-Congo Hemorrhagic Fever Virus (CCHFV)-Specific IgM and IgG antibodies in human sera using recombinant CCHFV nucleoprotein as antigen in $\mu$-capture and IgG immune complex (IC) ELISA tests. PLoS Negl Trop Dis. 2018;12:e0006366.

\section{Figures}


A

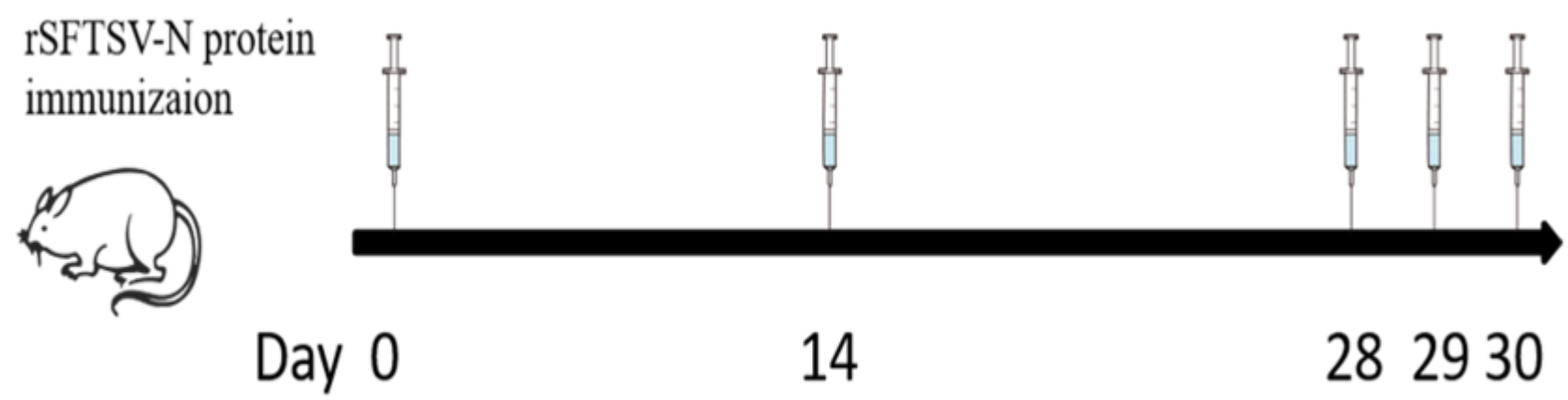

B

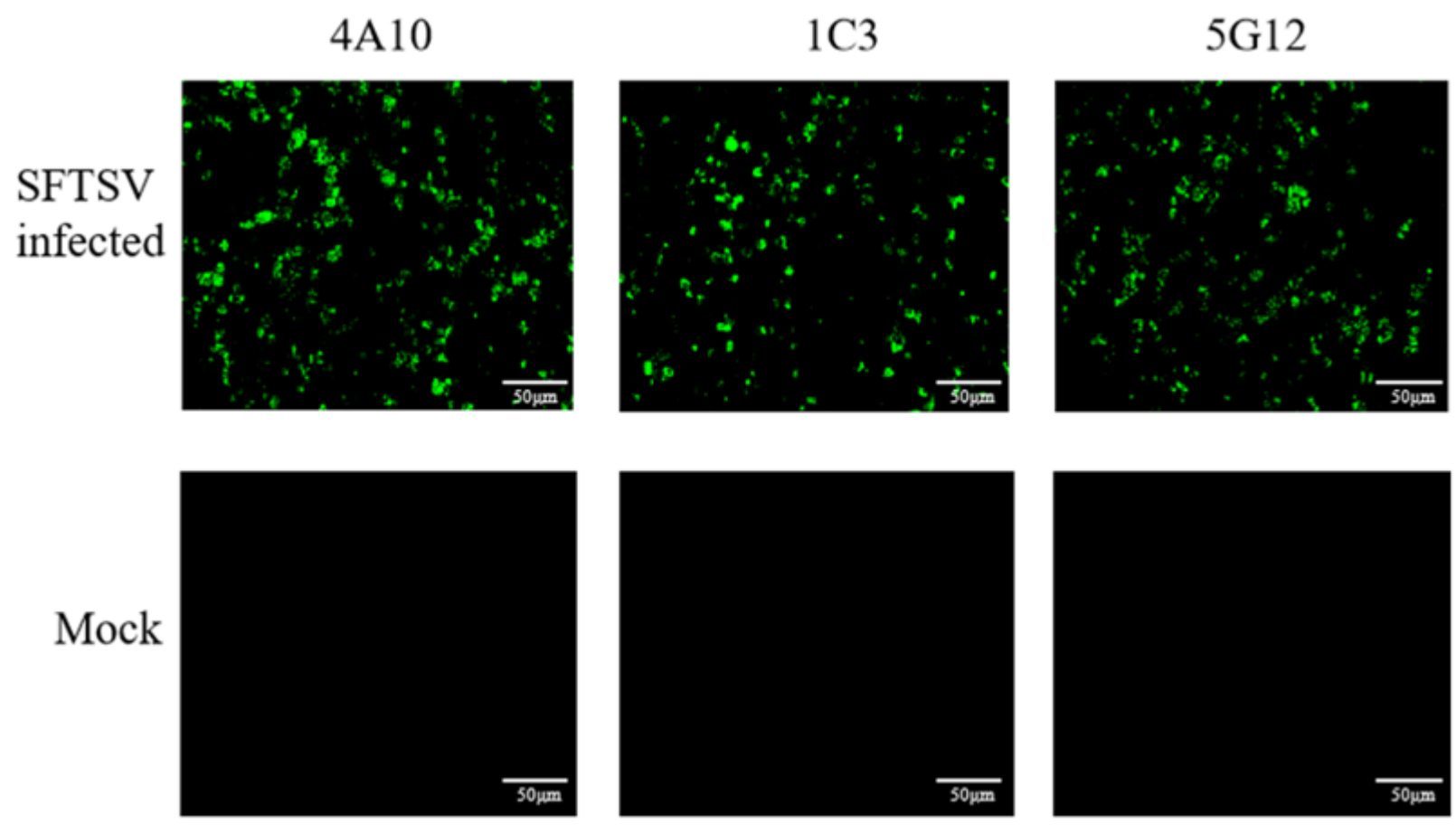

\section{Figure 1}

Preparation of monoclonal antibodies against rSFTSV-N protein. (A) BALB/C mice immune schedule. (B) Immunofluorescence analysis to verify the specificity of MAbs. SFTSV infected and mock Vero-E6 cells reacted with three MAbs and then detected by FITC labeled anti-mouse IgG and observed under an immunofluorescence microscope. 
A

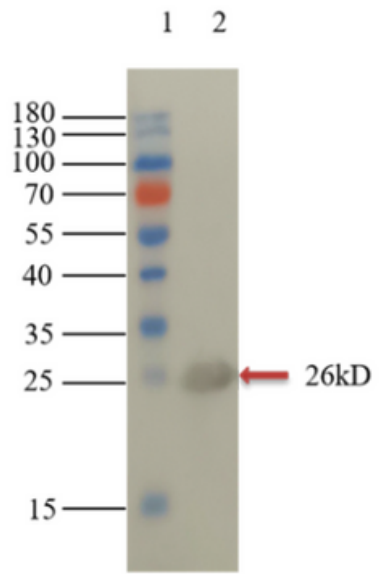

a

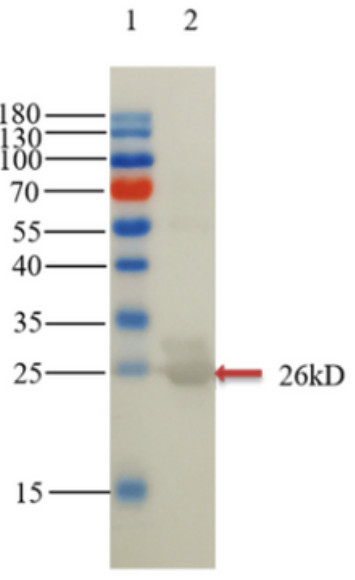

b

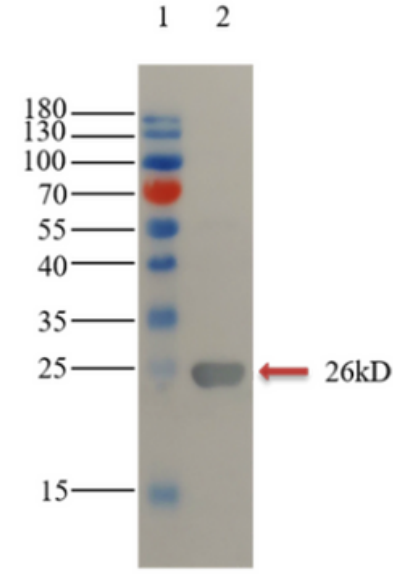

c

\begin{tabular}{|c|c|c|c|}
\hline Antibody type & $4 \mathrm{~A} 10$ & $1 \mathrm{C} 3$ & $5 \mathrm{G} 12$ \\
\hline $\mathrm{IgM}$ & - & - & - \\
\hline $\mathrm{IgG} 1$ & - & - & - \\
\hline $\mathrm{IgG} 2 \mathrm{a}$ & - & - & - \\
\hline $\mathrm{IgG} 2 \mathrm{~b}$ & + & + & + \\
\hline $\mathrm{IgG} 3$ & - & - & - \\
\hline $\mathrm{IgA}$ & - & - & - \\
\hline$\kappa$ & + & + & + \\
\hline$\lambda$ & - & - & - \\
\hline
\end{tabular}

Figure 2

Western blot ananysis and isotype detection of MAbs. (A) Western-blot analysis of MAbs against rSFTSVN protein. a: MAb 4A10; b: MAb 1C3; c: MAb 5G12. Lane 1: protein marker; lane 2: purified rSFTSV-N protein. (B) The antibody subclasses of three MAbs. The subclasses of the 3 MAbs were determined to be IgG2 $b$ and $k$ chain using a mouse MAb isotyping kit. 